\title{
The effectiveness of early intervention on malocclusion and its impact on craniofacial growth: A systematic review
}

Patrícia Valério ${ }^{1}$, (D) Tina Poklepović Peričić ${ }^{2}$, (D) Andrea Rossi ${ }^{3}$, (D) Cristina Grippaudo ${ }^{4}$, Júlia dos Santos Tavares Campos ${ }^{5}$, (D) Israel Júnior Borges do Nascimento ${ }^{6}$

\section{Highlights}

The early delivery of orthodontic treatment plays a critical role in craniofacial development and the establishment/re-establishment of appropriate stomatognathic function.
Early interventions effectively affect the masticatory system, increasing average bite force and general thickness of facial muscles.
This review emphasizes the need for well designed clinical trials that would consider patient-important outcomes.
${ }^{1}$ Institute of Biological Sciences, Federal University of Minas Gerais, Belo Horizonte, Minas Gerais, Brazil

Patricia V alerio Institute, Belo Horizonte, Minas Gerais, Brazil

${ }^{2}$ Department of Research in Biomedicine and Health, University of Split School of

Medicine, Split, Croatia

3 School of Dentistry, Università Cattolica del Sacro Cuore, and Fondazione Policlinico Universitario "A. Gemelli” IRCCS, Roma, Italy

4 School of Dentistry, Università Cattolica del Sacro Cuore, and Fondazione Policlinico Universitario "A. Gemelli” IRCCS, Roma, Italy

${ }^{5}$ Institute of Biological Sciences, Federal University of Minas Gerais, Belo Horizonte, Minas Gerais, Brazil

${ }^{6}$ School of Medicine, Federal University of Minas Gerais, Belo Horizonte, Minas Gerais, Brazil

School of Medicine, Medical College of Wisconsin, Milwankee, Wisconsin, United States of America

\section{Correspondence:}

Patricia Valerio Institute, Belo Horizonte, Minas Gerais, Brazil Email address:

patricia.valerio@,terra.com.br

Received: 31 May 2021

Accepted: 14 July 2021

Online First: 29 July 2021

\section{Abstract}

This review aims to assess the available evidence related to the effectiveness of early interventions on malocclusion and its impact on the craniofacial structure among children under six years of age. Furthermore, we aimed to evaluate the correlation between nutritive sucking behavior mechanisms on the oral facial components. We searched Medline, Embase, Cochrane Library, Scopus, and the LILACS from inception to December 10, 2020, to identify published randomized and non-randomized controlled trials that investigated the broad spectrum of early interventions for the treatment of malocclusions among pediatric patients under six years old. We have also included studies that evaluated the impact or the relationship between feeding alternatives, malocclusion, and craniofacial growth. Reviewers working in pairs investigators independently performed title and abstract screening, full-text screening, data extraction, risk of bias assessment using ROBINS-I tool, and rated the certainty of evidence using GRADE. Seven studies were included (783 patients), with an overall risk of bias classified as critical. Early treatment was shown to improve facial asymmetry, particularly in the lower part of the face, along with an increase of palatal volume and palatal surface. Early treatment showed important reduction of mandibular protrusion and length, leading to favorable sagittal growth of the maxilla. Furthermore, the early intervention significantly enhanced the average bite force magnitude (from $318.20 \mathrm{~N}$ to $382.79 \mathrm{~N}$ ) and increased the general thickness of facial muscles. Our findings suggest that the benefits generated by early orthodontic interventions are related to the improvement of craniofacial symmetry/bone structure, and refinement of masticatory ability and performance. Notwithstanding, there is still a need for further studies appraising patientimportant outcomes, such as quality of life and nutritional features.

Keywords: Bone Growth; Early Intervention; Malocclusion; Maxillofacial Development; Systematic Review 


\section{INTRODUCTION}

Oral diseases represent a highly relevant group of overall health-related conditions, affecting different age groups, genders, and socioeconomic classes. According to the World Health Organization (WHO), obtaining adequate oral health is the gateway to general health, well-being and overall quality of life. ${ }^{1}$

Malocclusion, defined as inappropriate or abnormal teeth alignment, as well as problematic craniofacial relationship, constitutes a major oral health issue worldwide. Its prevalence is ranging from $11 \%$ to $99 \%$, and may be associated with multiple factors, including genetic and environmental components. ${ }^{2-5}$

Among children with deciduous dentition, some conditions or childrens' habits may change the format and skeletal structure of facial bones, including 1 . the use of the pacifier, 2. finger sucking, and 3. traumatic injuries which result in an amorphous jaw, 4. atypical shaped or impacted teeth, and/or 5. parafunctional behavior. ${ }^{6-8}$

Recent evidence regarding the effectiveness of interventions of malocclusion has mostly referred to children older than six and involved multiple types of orthodontic appliances, like removable or fixed orthodontic devices. ${ }^{9,10}$ Notably, the presence of an altered maxilla-mandible relation might impair essential physiological functions, particularly on the temporary dentition. ${ }^{11-13}$ For instance, according to Woon et al. ${ }^{9}$ who included patients from seven to 12 years old, there is moderate evidence in favour of the short-term effectiveness of early treatment for malocclusion suggesting likely improvement in reverse overjet and/or in skeletal and soft tissue changes. There is, however, a lack of evidence on its long-term benefits. Similarly, Kallunki et al. ${ }^{10}$ have found some evidence that the early treatment of malocclusion, Class II, division 1, in particular, might lead to improved anteroposterior skeletal relationships. However, these systematic reviews and meta-analyses did not assess the impact of early interventions for malocclusion among children under six years of age, nor did they evaluate the relationship between treatment of malocclusion on temporary dentition and its impact on craniofacial growth. In addition, to the best of the author's knowledge, no published protocol nor systematic review (with or without meta-analysis) examining the influence of malocclusions on craniofacial development in children under six years of age has been performed. Therefore, this study aims to evaluate the influence of malocclusion on craniofacial development, especially in patients under six years of age, and to appraise the effects of early intervention on bone structure, as well as on functional traits. In addition, we aimed to evaluate studies assessing the impact or the relationship of feeding alternatives with malocclusions during childhood..

\section{METHODS}

\section{Registration and protocol}

This systematic review is reported following the Preferred Reporting Items for Systematic review and Meta-analyses (PRISMA) and was registered in the PROSPERO (CRD42021226182). ${ }^{14}$ The supplementary materials containing the list of excluded studies (with reasons) and the list of studies awaiting classification are available in Appendix 1.

\section{Sources of information, research strategy, and selection of studies}

On December 10, 2020, we searched five primary electronic scientific databases (Embase, The Cochrane Library, MEDLINE, Scopus, and LILACS) to identify studies investigating the association between malocclusion and associated interventions and craniofacial growth. The search strategy was created in collaboration between the dental specialists from the group and an information specialist based at the Federal 
University of Minas Gerais, Brazil. Besides, we looked for ongoing clinical trial registrations on ClinicalTrials.gov. The search strategy for all aforementioned databases is available in Appendix 2.

Reviewers (PV, TPP, AR, CG, JSTC, and IJBN) working in pairs, independently performed title and abstract screening as well as full-text analysis. In addition, the investigators independently performed data extraction, and bias assessment risk. Decision conflicts were resolved by consulting a third independent author (PV).

\section{Eligibility, inclusion and exclusion criteria}

The eligibility criteria were developed based following the well-established PICOS acronym, as follows: 1. Participants: pediatric patients (defined as < six years old), requiring mandatory or elective oral evaluation and who were diagnosed with malocclusion. We excluded patients diagnosed with any syndromic disease, patients older than six years old, and those reportedly using hormonal therapy; 2. Interventions: a broad spectrum of interventions, including, but not limited to, selective grinding, direct tracks, functional appliance, hard food features, or myotherapy. We decided to include studies evaluating the impact or the relationship of feeding alternatives with malocclusion, as they directly interfere with oral occlusion characteristics during childhood; 3. Comparator or control group: we considered any head-to-head comparison along with any control or no intervention comparator, regardless of the presence or not of co-interventions and adjuvant therapies; 4. Outcomes: tridimensional symmetry and proportionality features and masticatory functionality; 5. Study design: randomized or quasi-randomized controlled trials, cluster and cross-over trials, and observational studies were eligible for inclusion. It is worthwhile stating that if a study enrolled patients both within and outside our pre-established target population, i.e., pediatric patients up to six years old and those above six years old, we attempted to contact the authors of the study in order to retrieve a complete dataset for each age group and include only the data concerning children that met our inclusion criteria. The attempt to contact the studies' authors was made by email, at least two times, between February/2021 to April/2021. However, if the corresponding authors of any study with the aforementioned issue did not reply to the review team, we included the study but prominently decreased the certainty of evidence and highlighted this limitation when describing the characteristics of the included studies.

\section{Data items and collection}

We extracted primary data from eligible studies including author's name, publication year, journal's name and impact factor, origin country, along with the type of intervention used in the study, length of intervention and follow-up, method used for diagnosing malocclusion, sample characteristics, primary and secondary outcomes. Participants' age or age range, overall summary of the effect related to the proposed intervention, besides crucial studies' limitations were also extracted.

We planned to perform our statistical analysis using the Review Manager 5 (RevMan 5), using a random-effects model for combining data because of variation in the interventions. However, because of the lack of similarity between studies, the meta-analyses were not performed.

\section{Risk of bias in individual studies and evaluation of the level of evidence}

Risk of bias assessment was performed using the Risk of Bias In Non-randomized Studies of Interventions (ROBINS-I) tool, which considers seven domains. ${ }^{15}$ Each domain is judged with either "low risk", "moderate risk", "serious risk", "critical risk" or as "no information", when no 
specific information is provided. The certainty of the evidence was rated using the adapted GRADE approach, as we did not quantitatively pooled obtained results. ${ }^{16}$ We assessed the certainty of evidence for the primary outcomes by considering risk of bias in the studies, inconsistency, imprecision, indirectness, and a possibility for publication bias. For those studies including patients within and outside our established target population, we downgraded the domain of indirectness.

\section{RESULTS}

We initially identified 8,591 publications, of which 1,949 were duplicates. Most publications $(6,533)$ were excluded during the title and abstract screening.
Regarding the 109 studies identified during fulltext analysis, 23 publications could not be retrieved (classified as "awaiting classification"), 78 were excluded, with reasons provided, and seven studies were included in the systematic review (Figure 1). ${ }^{17-23}$ Additional results can be accessed in the supplementary results section (Appendix 3).

\section{Characteristics of included studies}

We present the summary characteristics of the seven included studies in Table 1. Studies were published in five different journals with journal impact factors varying from 0.79 to 2.2. All included studies were prospective cohort studies, with patients from various geographic contexts (Slovenia, Brazil, Italy, Argentina, and Japan). The oldest study was published in 1996, while the most recent one was published in 2013.

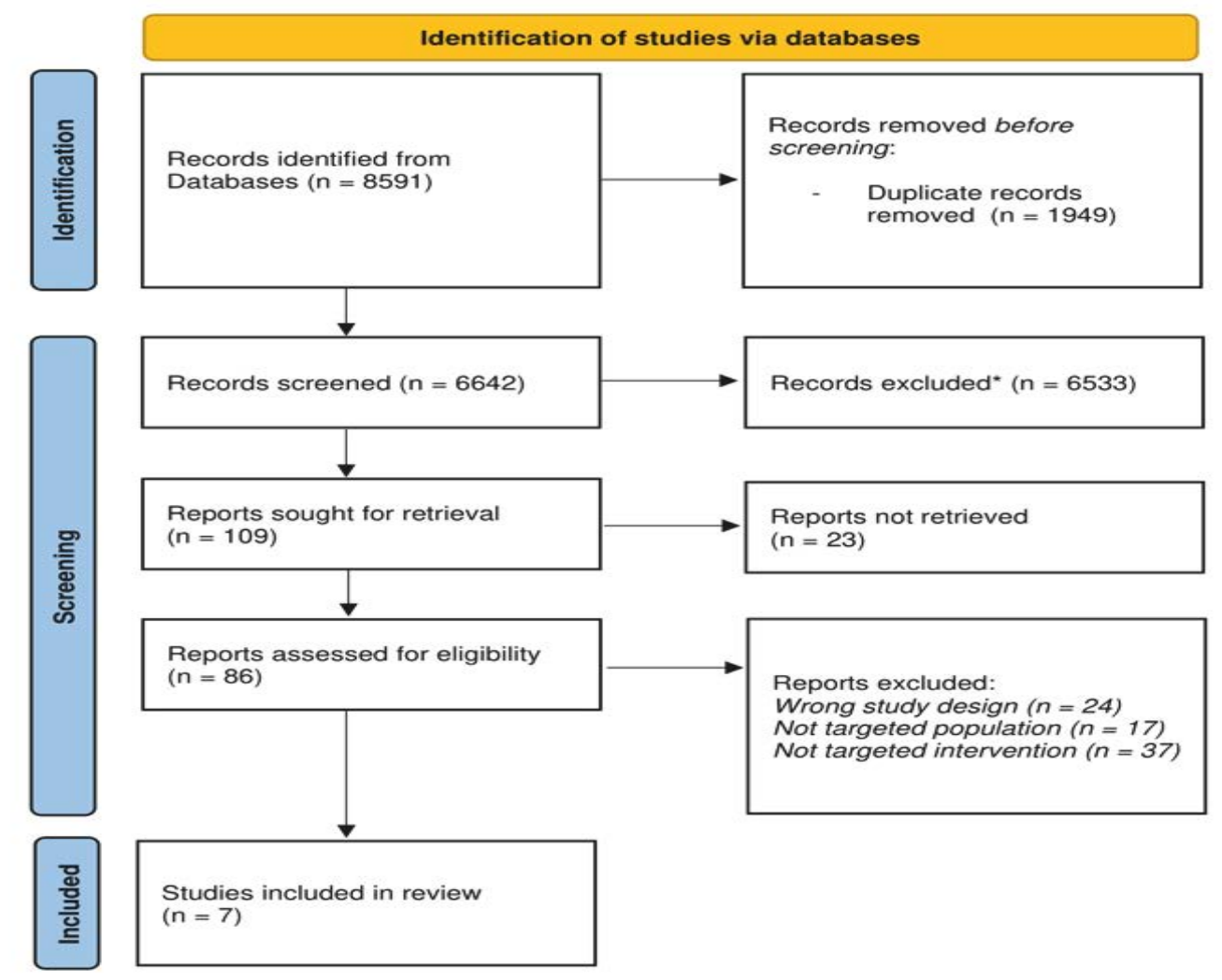

* Records excluded by a human and not by an automation tool.

Figure 1. PRISMA 2020 flow diagram for new systematic reviews 
Table 1. Summary characteristics of included studies

\begin{tabular}{|c|c|c|c|c|c|c|c|c|c|}
\hline $\begin{array}{l}\text { First Author } \\
\text { Surname }\end{array}$ & $\begin{array}{l}\text { Publication } \\
\text { Year }\end{array}$ & Journal Name & $\begin{array}{l}\text { Journal } \\
\text { Impact } \\
\text { Factor* }\end{array}$ & $\begin{array}{l}\text { Origin } \\
\text { Country and } \\
\text { Setting }\end{array}$ & Study Design & Main Objective & $\begin{array}{l}\text { Number of } \\
\text { Included } \\
\text { Patients }\end{array}$ & Patients Age & Age Range \\
\hline Primozic et al & 2013 & $\begin{array}{l}\text { European Journal } \\
\text { of Orthodontics }\end{array}$ & 2.2 & Slovenia & $\begin{array}{l}\text { Longitudinal } \\
\text { Cohort Study }\end{array}$ & $\begin{array}{l}\text { To assess whether correction of } \\
\text { unilateral posterior crossbite in the } \\
\text { primary dentition results in } \\
\text { improvement of facial symmetry and } \\
\text { increase of palatal surface area and } \\
\text { palatal volume }\end{array}$ & 60 & $\begin{array}{l}\text { Mean age } \\
(5.2 \pm 0.7)\end{array}$ & 3.4 to 6.7 \\
\hline Castelo et al & 2010 & $\begin{array}{l}\text { Journal of } \\
\text { Clinical Pediatric } \\
\text { Dentistry }\end{array}$ & 0.79 & Brazil & $\begin{array}{l}\text { Longitudinal } \\
\text { Cohort Study }\end{array}$ & $\begin{array}{l}\text { To determine morphological and } \\
\text { functional effects on masticatory system } \\
\text { of early treatment of functional } \\
\text { posterior crossbite in young children }\end{array}$ & 23 & Not available & 5 to 6 \\
\hline Tollaro et al & 1996 & $\begin{array}{l}\text { American Journal } \\
\text { of Orthodontics } \\
\text { and Dentofacial } \\
\text { Orthopedics }\end{array}$ & 1.96 & Italy & $\begin{array}{l}\text { Longitudinal } \\
\text { Cohort Study }\end{array}$ & $\begin{array}{l}\text { To evaluate the effects of a functional } \\
\text { appliance on the craniofacial skeleton in } \\
\text { children with Class III malocclusions }\end{array}$ & 30 & $\begin{array}{l}\text { Mean age } \\
(5.64 \pm 1.1)\end{array}$ & Not available \\
\hline $\begin{array}{l}\text { Lescano de Ferrer et } \\
\text { al }\end{array}$ & 2006 & $\begin{array}{l}\text { Revista de la } \\
\text { Facultad de } \\
\text { Ciencias Medicas } \\
\text { (Argentina) }\end{array}$ & $\begin{array}{l}\text { Not } \\
\text { available }\end{array}$ & Argentina & $\begin{array}{l}\text { Longitudinal } \\
\text { Cohort Study }\end{array}$ & $\begin{array}{l}\text { To show the occlusal characteristics of } \\
\text { children in the Córdoba, Argentina and } \\
\text { to show favorable effects provided by } \\
\text { breastfeeding }\end{array}$ & 290 & 5 & Not available \\
\hline Carrascoza et al & 2006 & $\begin{array}{l}\text { Jornal de } \\
\text { Pediatria (Brazil) }\end{array}$ & 2.02 & Brazil & $\begin{array}{l}\text { Longitudinal } \\
\text { Cohort Study }\end{array}$ & $\begin{array}{l}\text { To identify and assess the possible } \\
\text { consequences of bottle-feeding on the } \\
\text { oral facial development } \\
\text { of children who were breastfed up to at } \\
\text { least six months of age }\end{array}$ & 202 & 4 & Not available \\
\hline Kajiyama et al & 2004 & $\begin{array}{l}\text { American Journal } \\
\text { of Orthodontics } \\
\text { and Dentofacial } \\
\text { Orthopedics }\end{array}$ & 1.96 & Japan & $\begin{array}{l}\text { Longitudinal } \\
\text { Cohort Study }\end{array}$ & $\begin{array}{l}\text { To investigate the effects of the } \\
\text { maxillary protractor bow appliance on } \\
\text { dentoalveolar structure and skeletal } \\
\text { morphology in patients with Class III } \\
\text { malocclusions in different dental stages }\end{array}$ & 120 & $\begin{array}{l}\text { Mean age } \\
(5.0 \pm 10 \\
\text { months) for } \\
\text { deciduous } \\
\text { dentition patients }\end{array}$ & Not available \\
\hline $\begin{array}{l}\text { Primozic and } \\
\text { Ovsenik et al }\end{array}$ & 2009 & $\begin{array}{l}\text { European Journal } \\
\text { of Orthodontics }\end{array}$ & 2.2 & Slovenia & $\begin{array}{l}\text { Longitudinal } \\
\text { Cohort Study }\end{array}$ & $\begin{array}{l}\text { To assess facial asymmetry and palatal } \\
\text { volume (pre- and post-treatment) in two } \\
\text { groups of children, one with a unilateral } \\
\text { crossbite and the other with no } \\
\text { crossbite }\end{array}$ & 58 & $\begin{array}{l}\text { Mean age } \\
(4.9 \pm 0.98)\end{array}$ & 3.6 to 6.6 \\
\hline
\end{tabular}

* Impact Factor derived from the Scientific Journal Ranking Database 
Overall, 783 pediatric patients were included, with the lowest age being three years. As far as the main objectives, studies were mainly designed to assess: 1. The impact of early interventions in facial symmetry and bone structure or morphology ${ }^{17,19-21} ; 2$. The effect of early treatment on the masticatory system and functional traits 22 ; and 3. The correlation between malocclusion features and potential feeding preferences (either through natural feeding [breastfeeding] or artificial alternatives [cup-feeder and bottle-feeder]). ${ }^{18,23}$ Baseline characteristics of included patients are presented in Table 2. In general, most included patients had primary dentition and were frequently healthy and were diagnosed (clinically or radiologically) with multiple categories of malocclusion (anterior and posterior crossbite, midline deviation, or class III malocclusion).

\section{Types of interventions and length of intervention}

Table 2 shows a complete description of the included studies' interventions. Overall, various kinds of interventions have been described in the included studies to treat pediatric patients' malocclusion. Among the studies that evaluated the impact of early interventions in craniofacial morphology, masticatory system, functional traits, or dentoalveolar structures, typical treatment included acrylic plate to expand the maxillary arch, and removable maxillary expansion plates (with a maximum length of time of two years). For the studies evaluating the effect of specific nutritive sucking behavior mechanisms on the oral facial components, one study compared regular breastfeeding to bottle-feeding, while another study compared cup-feeding with bottlefeeding (both artificial nipples).

\section{Summary of findings and intervention effects}

Table 3 provides the summary of main findings in relation to the primary outcomes described in the included studies.

\section{The effect of early interventions on craniofacial morphology and bone structure}

In four studies ( $\mathrm{n}=153$ participants), the primary outcome included morphological and bone structure changes after early therapeutic interventions in pediatric patients. ${ }^{17,19-21}$ Interestingly, two studies ${ }^{20,21}$ presented statistically significant results showing that children with a prior diagnosis of malocclusion had more significant facial asymmetry, especially in the lower part of the face compared to children that were not diagnosed with malocclusion. Additionally, both studies stated a remarkable improvement of facial symmetry, more prominently in the lower facial area after the delivery of the appropriate treatment. Furthermore, these studies had also recognized a decreased palatal volume and palatal surface area among individuals with malocclusion. Still, the use of early interventions against malocclusion promoted a significant increase in this parameter. One study, based on craniofacial skeletal characteristics (linear and angular measurements), evidenced that early functional treatment of Class III malocclusion substantially interferes on linear measurements, providing an essential anterior morphogenetic rotation of the mandible (therefore, reducing mandibular protrusion and length) along with leading to favorable sagittal growth of the maxilla. ${ }^{19}$ One study assessed the effect of dental appliances on dentoalveolar structures and skeletal morphology in different dental stages. ${ }^{17}$ In short, treating patients with deciduous dentition showed statistically significant higher mean bony and dentoalveolar changes than those with mixed dentition, the analysis was shown by 3-way ANOVA, which presents a significant effect for 2 factors when the authors compared the types of dentition. 
Table 2. Detailed description of interventions used in included primary studies

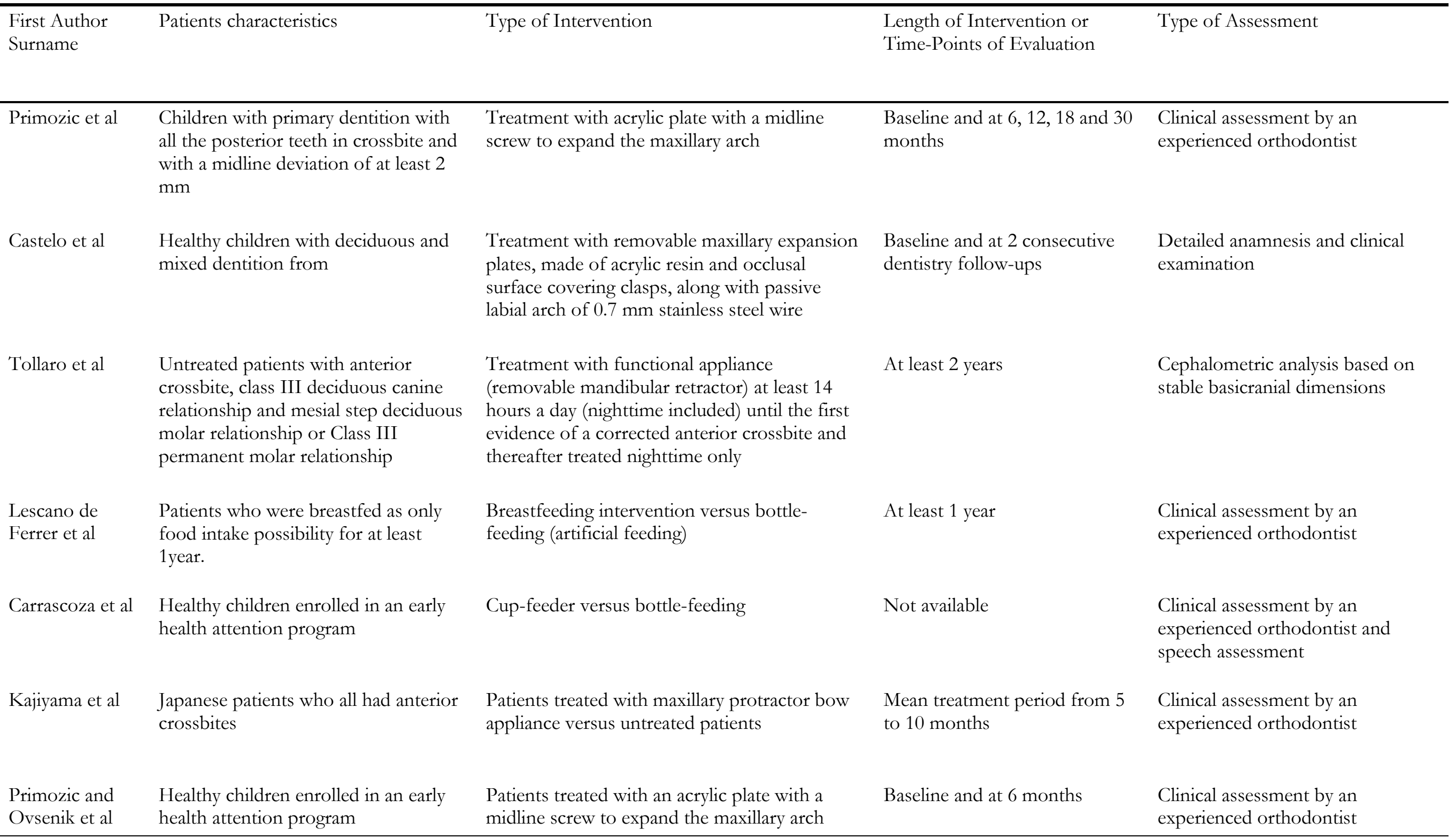


Table 3. Summary of results among included studies

\begin{tabular}{ll}
\hline $\begin{array}{l}\text { First Author } \\
\text { Surname }\end{array}$ & Main Outcomes \\
\hline Primozic et al & $\begin{array}{l}\text { Facial Asymmetry, Palatal Surface Area } \\
\text { and Palatal Volume }\end{array}$
\end{tabular}
and Palatal Volume

Assessment Tools and Methods

For all outcomes, Konica and Minolta Vivid 910 Laser

Scanners were used to evaluate patients. Images were imported into Reverse Modelling Software (Rapidform, 2006)

Castelo et al

Maximal bite force, ultrasonographic masticatory muscle thickness and facia asymmetry

Tollaro et al

Craniofacial skeleton features (Linear measurements for the assessment of sagittal relationship, mandibular dimensions, and angular measurements for the assessment of cranial base angulation, vertical relationship and of mandibular ramus and condyle inclinations)

Presence and type of malocclusion, dysfunctional oral habits and lingual interposition
Masseter and Temportalis Muscle Thickness were assessed by ultrasonography, while bite force was evaluated through a pressurized and flexible tubes connected to a sensor element. Facial asymmetry was assessed by frontal photographs

Lateral cephalometric analysis based on stable base cranial dimensions

Clinical assessment for all outcomes
Summary of Findings or Intervention Effect

Children with malocclusion had statistically significantly greater facial asymmetry in the lower part of the face $(\mathrm{P}<0.05)$ and a significantly smaller palatal volume $(\mathrm{P}<0.05)$ than the children without malocclusion at baseline. Among the group treated for malocclusion, there were an increase in the average distance of the upper part of face and in the overlapping of the lower part of the face were observed $(\mathrm{P}<0.05)$. There were no statistically significant differences between the two groups at 6-, 12-, 18-, and 30 -months follow-ups. Treatment of unilateral posterior crossbite in the primary dentition period resulted in an improvement of facial symmetry in the lower part of the face $(\mathrm{P}<0.05)$ and increase of the palatal surface area and palatal volume $(\mathrm{P}<$ $0.001)$.

The average bite force magnitude increased significantly among treated patients. The thickness of the temporalis at rest and in the maximal intercuspal position showed significant increase during the time points in comparison with baseline characteristics among included patients, whereas the masseter thickness showed no differences among the stages. The correlation between the angle of the eye and the angle of the mouth in relation to the mid-sagital plane increased from baseline to final check-up appointment.

Linear measurements (A-Vert, Pr-Vert and Go-Vert) exhibited significant larger increments in treated patients, while B-Vert T, Id-Vert T, Pg-VertT evidenced significantly smaller increment in treated groups. The main significant findings in the treated group were an anterior morphogenetic rotation of the mandible as a result of an upward-forward direction of condylar growth, a more vertical orientation of the ramus, and a reduced gonial angle; reduced mandibular protrusion and total length; increased maxillary protrusion; increased maxillary dentoalveolar protrusion and reduced mandibular dentoalveolar protrusion.

Patients who were breastfed showed high percentage of normal occlusion $(69.1 \%)$ while children who received artificial feeding had higher percentage of malocclusion. In the breastfed group, typical types of malocclusion were crossbite and open bite. No anterior crossbite was observed among breastfed patients. Breastfed patients significantly differed from artificial fed subjects regarding the presence of lateral crossbite. With regards to dysfunctional oral habits, nocturnal respiratory insufficiency was higher among patients bottle-fed. As for dysfunctional oral habits, the highest percentage found was related lo lingual interposition associated lo open biting, presented by children who had been bottle-fed. 


\section{Table 3. Continued}

Carrascoza et al Presence and type of malocclusion, muscle aspects, articulation characteristics, breathing patterns, palate depth, maxillary arch shape and face symmetry

Kajiyama et al

Craniofacial skeleton features (Linear and angular measurements)

Primozic and Ovsenik et al
Clinical assessment for all

\section{outcomes}

Lateral cephalograms before and after treatment to compare the dentofacial change
Lip closure was observed in $65 \%$ of bottle users and in $82 \%$ of cup users $(p=0.0065)$. With regard to the tongue resting place, among the children who used cups, $73 \%$ presented tongue resting in the maxillary arch (desirable). Among the children who used feeding bottles, $53 \%$ presented tongue resting in the mandibular arch or between the arches (a change from normality), revealing hypotonicity of tongue muscles $(\mathrm{p}<0.0001)$. There was greater occurrence of nose breathing among children using cups $(69 \%)$. Among those who used a bottle, $63 \%$ presented mouth or mixed breathing $(\mathrm{p}<0.0001)$. The shape of the maxillary arch was different in the two groups, maxillary atresia being present in $22 \%$ of the bottle users and in $10 \%$ of the cup users $(p=0.0206)$. There was no difference between the groups in terms of malocclusion, articulation, palate depth and presence of facial asymmetry $(\mathrm{p}>0.05)$.

The mechanisms of improving anterior crossbite were similar in both groups; however, the mean skeletal and dentoalveolar changes in the deciduous dentition group were significantly greater than those in the mixed dentitions group. The clinical effects of maxillary protractor bow appliance treatment were greater in the deciduous-dentition group than in the early-mixed-dentition group. Marked forward displacement of the maxillary structures was achieved as an outcome of early treatment in the deciduous dentition group, whereas the mixed dentition group showed only a small measurable maxillary advancement. In addition, clockwise relocation of the mandible was shown more significantly in the deciduous dentition group than in the mixed dentition group and Rapid correction of anterior crossbite in the deciduous dentition group was attributed to the high reduction of ANB angle with maxillary down and forward movement and mandibular clockwise relocation. Lastly, although the mechanisms of improving anterior crossbite were similar in both groups, the mean skeletal changes in the deciduous dentition group were greater than those in the mixed dentition group. These findings indicate that early treatment of Class III malocclusion seems to induce more favorable overall craniofacial changes than late treatment.

The children with crossbite had statistically significantly greater asymmetry of the face $(\mathrm{P}=0.042)$, especially the lower third $(\mathrm{P}=0.039)$, and a significantly smaller palatal volume $(\mathrm{P}=0.045)$ than the normal subjects at baseline. There were no statistically significant differences between the two groups at 6 months of follow-up. Treatment of a crossbite in the primary dentition corrected the facial asymmetry, particularly the lower part of the face The palatal volume of the crossbite children increased as a result of orthodontic intervention to similar levels exhibited by the normal children. Palatal volumes and increases in palatal volume from baseline to late follow-up. Palatal volume was greater in the control group compared with the crossbite group at baseline. However, this difference was greatly reduced after treatment. The increase in palatal volume was statistically

significantly greater in the crossbite patients compared with the control group. surface facial images usir images using t Reverses were impotc Modelling Sof

(Rapidform). Dental cast volu was scanned at a distance of $60 \mathrm{~cm}$ with the same laser scanner using lens with a focus distance of 25 $\mathrm{mm}$ 


\section{The effect of early interventions on the masticatory system and functional traits}

One study ( $\mathrm{n}=23$ patients) specifically associated with the analysis of early interventions on the masticatory system and functional traits. ${ }^{22}$ According to Castelo et al. ${ }^{22}$, the implementation of an early treatment protocol for patients with deciduous dentition significantly increased the average bite force magnitude (initial bite force of $318.20 \mathrm{~N}$ and final bite force of $382.79 \mathrm{~N}$ ). In addition, the delivery of an early intervention increased the thickness of temporalis muscle at rest, whereas the masseter thickness did not differ at any evaluated stages. Furthermore, an improvement in facial proportionality was observed, characterized by the increase between the angle of the eye and the angle of the mouth in relation to the mid-sagittal plane.

\section{The correlation between malocclusion features and potential feeding preferences}

Two studies ( $\mathrm{n}=492$ individuals) focused on the relevant correlation between malocclusion and infant feeding categories. ${ }^{18,23}$ Based on a populational cohort in Argentina, one study ${ }^{23}$ showed a higher percentage of normal occlusion among individuals breastfed than those under artificial feeding methodology. Furthermore, the study evidenced that bottle-fed patients had a higher incidence of dysfunctional oral habits and nocturnal respiratory insufficiency. Similarly, one Brazilian research assessing the possible consequences of bottle-feeding on oral-facial development among children breastfed for at least six months has shown that cup-fed children presented a higher percentage of the tongue resting in the maxillary arch (desirable position). ${ }^{18}$ Additionally, $63 \%$ of included individuals had a mouth or mixed breathing (an undesirable and inadequate type of breathing). There was also a high incidence of maxillary atresia among patients in the study (a mean prevalence of $16 \%$ in the two bottle-feeding categories).

\section{Risk of bias assessment}

Table 4 shows the risk of bias assessment for the seven included studies. Based on ROBINS-I criteria, validity of the results from all eight primary included studies was rated as "critical" (Table 4). Common methodological drawbacks were the inexistence of a published study protocol, the absence of reports on patients' baselines characteristics, , potential issues regarding standardized delivery of intervention for an exact subset of patients, the unavailability of information about patient dropouts, and the inability of knowing whether patients were previously selected from a major group of patients in a specific medical facility.

\section{Certainty of evidence - GRADE assessment}

Table 5 shows the overall certainty of evidence using the GRADE approach for the important outcomes. The GRADE level of certainty of the evidence was very low for facial symmetry, masticatory system, and functional traits. We downgraded by two levels the correlation between malocclusion and feeding preferences due to study design limitations and risk of bias. 
Table 4. ROBINS-I assessments

\begin{tabular}{|c|c|c|c|c|c|c|c|c|}
\hline $\begin{array}{l}\text { First Author } \\
\text { Surname }\end{array}$ & D1 & $\mathrm{D} 2$ & D3 & D4 & D5 & D6 & D7 & Overall \\
\hline Primozic et al & Low & Low & Low & Low & Low & Critical & No Information & Critical \\
\hline Castelo et al & Critical & Critical & Low & Low & No Information & Critical & No Information & Critical \\
\hline Tollaro et al & Low & No Information & Low & Low & No Information & Critical & No Information & Critical \\
\hline $\begin{array}{l}\text { Lescano de } \\
\text { Ferrer et al }\end{array}$ & .Low & Low & Low & Low & No Information & Critical & No Information & Critical \\
\hline Carrascoza et al & Low & Low & Low & Low & No Information & Critical & No Information & Critical \\
\hline Kajiyama et al & Low & Low & Low & Low & No Information & Critical & No Information & Critical \\
\hline $\begin{array}{l}\text { Primozic and } \\
\text { Ovsenik et al }\end{array}$ & Low & Low & Low & Low & Low & Critical & No Information & Critical \\
\hline $\begin{array}{l}\text { D1 - Bias due to } \\
\text { D2 - Bias due to } \\
\text { D3 - Bias in class } \\
\text { D4 - Bias due to } \\
\text { D5 - Bias due to } \\
\text { D6 - Bias in meas } \\
\text { D7 - Bias in selec }\end{array}$ & $\begin{array}{l}\text { lection of } \\
\text { ication of } \\
\text { eviations } f \\
\text { issing dat } \\
\text { rement of } \\
\text { on of the }\end{array}$ & interventions & & & & & & \\
\hline
\end{tabular}


Certainty of the evidence (GRADE)

\begin{tabular}{|c|c|c|c|c|c|c|c|c|}
\hline Outcome & $\begin{array}{l}\text { Study population } \\
\text { and study design }\end{array}$ & Summary effect & $\begin{array}{l}\text { Methodological } \\
\text { Limitations* }\end{array}$ & Inconsistency† & Indirectness $\ddagger$ & Imprecision $\$ & $\begin{array}{l}\text { Publication } \\
\text { bias }\end{array}$ & Overall Quality \\
\hline $\begin{array}{l}\text { Craniofacial } \\
\text { morphology and } \\
\text { bone structure }\end{array}$ & $\begin{array}{l}4 \text { studies (153 } \\
\text { patients) }\end{array}$ & $\begin{array}{l}\text { Potential } \\
\text { improvement on } \\
\text { facial symmetry and } \\
\text { increase of palatal } \\
\text { surface area and } \\
\text { palatal volume }\end{array}$ & Critical & Not serious & Not serious & Serious & Not serious & $\begin{array}{l}\bigoplus O O O \\
\text { VERY LOWa }\end{array}$ \\
\hline $\begin{array}{l}\text { Masticatory system } \\
\text { and functional trait }\end{array}$ & $\begin{array}{l}1 \text { study (23 } \\
\text { patients) }\end{array}$ & $\begin{array}{l}\text { Increased maximal } \\
\text { bite force and } \\
\text { enhanced masticatory } \\
\text { muscle thickness }\end{array}$ & Critical & Not serious & Not serious & Serious & Not serious & $\begin{array}{l}\oplus \circ O \bigcirc \\
\text { VERY LOWb }\end{array}$ \\
\hline $\begin{array}{l}\text { Impact of feeding } \\
\text { preferences }\end{array}$ & $\begin{array}{l}2 \text { studies (492 } \\
\text { patients) }\end{array}$ & $\begin{array}{l}\text { Higher prevalence of } \\
\text { malocclusion among } \\
\text { patients undergoing } \\
\text { artificial feeding }\end{array}$ & Critical & Not serious & Not serious & Not serious & Not serious & $\begin{array}{l}\oplus \circ O \bigcirc \\
\text { VERY LOWc }\end{array}$ \\
\hline
\end{tabular}

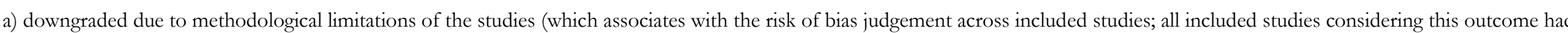
an overall critical risk of bias mainly due to measures of the assessed outcome, which could be potentially interfered by the examiner and by inter-rater evaluation), and imprecision (total number of enrolled patients must be considered insufficient, taking into account malocclusion's prevalence and distribution across populations)

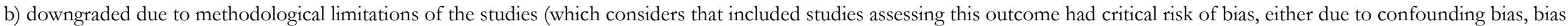
due to selection of participants, or due to measures of outcome), and imprecision (insufficient number of enrolled individuals to make definite conclusions)

c) downgraded due to methodological limitations of the studies (primarily because all included studies considering this outcome had overall critical risk of bias)

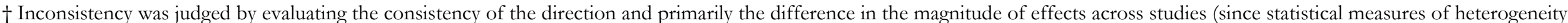
are not available). As we did not find differing results for each outcome across included studies, we considered "not serious" risk for inconsistency

$\ddagger$ We did not downgrade the indirectness domain because all conclusion and analyses made by included studies were equivalent between research evidence and the clinical context

$\int$ We downgraded the imprecision domain for the two first outcomes because they included a limited number of participants

I We downgraded the publication bias domain if the body of literature appears to evidence a limited number of positive effects or when studies are reported in trial registries but not published.

\section{GRADE Working Group grades of evidence}

High certainty: we are very confident that the true effect lies close to that of the estimate of the effect.

Moderate certainty: we are moderately confident in the effect estimate; the true effect is likely to be close to the estimate of the effect, but there is a possibility that it is substantially

different.

Low certainty: our confidence in the effect estimate is limited; the true effect may be substantially different from the estimate of the effect.

Very low certainty: we have very little confidence in the effect estimate; the true effect is likely to be substantially different from the estimate of effect. 


\section{DISCUSSION}

The presented systematic review assessed the relationship of early intervention on temporary dentition malocclusions, and the impact of these interventions on facial bone structures is the first comprehensive analysis including patients under six years old. Based upon a considerable number of representative databases and including 783 patients from different settings, there is evidence that there may be positive effects of early interventions on facial symmetry, palatal volume improvement, and craniofacial skeletal characteristics, with very low certainty of evidence. Furthermore, included studies showed an increase on average bite force magnitude and facial muscle thickness modification, representing actual results on the masticatory system and functional traits. Lastly, it has been perceived that pediatric individuals who underwent artificial feeding were more likely to have dysfunctional oral habits and respiratory insufficiency, potentially related to the manifestation of malocclusion issues. Overall, our results provide relevant insights for orthodontists, pedodontists, general dentists, functional orthopedists, and policymakers to design and develop specific oral care for pediatric patients. Also, we highlighted quantitative information for dentistry professionals regarding strategic treatment planning. Nevertheless, it is worthwhile mentioning that

Taking into account the results obtained in our systematic review, some researchers may still argue against the observations of improvements in facial symmetry, palatal volume, and craniofacial skeletal characteristics, considering that the craniofacial growth is expected to progress based upon its pre-existing genetic determinants, which will overtake any intervention or exposure. Thus, any improvement achieved in an early stage of an orthodontic intervention is unlikely to have long-lasting effects. However, it is crucial to remember that genetic predisposition to malocclusion can be altered by epigenetic factors, which change not only the patient's phenotype, but also the genotype of bone cells submitted to a specific stimuli. ${ }^{24-29}$

We did not evaluate a pooled effect for any specific outcome, because most included studies performed general and descriptive analyses of achieved results. Furthermore, there was a significant variation among study protocols, associated interventions, and participants' primary characteristics. Thus, it would not be appropriate to evaluate in a meta-analysis those results. However, as proposed by Campbell et al. ${ }^{30}$, we provided a categorical-specific description of correlated results to guide researchers with the synthesis prepared.

We found a considerable number of positive effects associated with the application of early interventions on malocclusions and the interference on craniofacial morphology and bone structure. ${ }^{31}$ Overall, subjects who receive early treatment have a more extraordinary ability to modify skeletal growth (either in symmetric or structural features) and to improve patient selfesteem and satisfaction with therapy. Moreover, it is believed that these interventions might also reduce the potential for iatrogenic dental injury (trauma, root resorption, or decalcification). ${ }^{32,33}$ The achievement of these critical patient-related outcomes is pertinent in the contemporary world because young children with aesthetic-related dental deformities are easy and probable targets for bullies. Therefore, oral aesthetics can severely interfere with children's quality of life, resulting in physical, social, and psychological impairment. ${ }^{34}$ Additionally, we found a remarkable demonstration that the correction of malocclusion in early life stages might objectively provide positive effects in mastication, deglutition, and other stomatognathic functions, strengthening the rationale of cosmetics purposes. Furthermore, it is believed that patients who adhere to phase I interventions (started during 
primary or early transitional dentition) have better and more stable results considering a long period of analysis. Also, previous studies have suggested that specific types of malocclusion might be more susceptible to more significant beneficial effects than other types of occlusion problems.

While planning the execution of the present review, we initially prioritized six significant primary outcomes for assessment in the study. Noticeably, the evaluation of facial symmetry and associated achievement of better aesthetic level, proportionality features, along with masticatory functionality was common outcomes evaluated amid included studies. In fact, these agreedstandardized sets of outcomes should be measured and reported in all clinical trials, especially in healthcare-related areas, classifying them as relevant "core outcome set". ${ }^{35}$ However, other critical patient-related outcomes, such as improvement of the patient's quality of life and potential nutritional and food intake characteristics attributed to the prior presence of malocclusion, were not considered in any of the eight included studies. Therefore, we emphasize and advocate the need for an enhanced design of future clinical trials in terms of considering patient-important outcomes, putting the patient at the center of medical decision-making. This might reflect on how a patient feels, functions, and survives with the condition in analysis. ${ }^{36}$

Some authors ${ }^{37-39}$ have already endorsed theories that the best period for starting orthodontic interventions against malocclusion might be during the musculoskeletal peak of growth (typically at 10-11 years old [females] and 12-13 years old [males]). Interestingly, this theory's principal proponents believe that an early intervention against malocclusion might predispose traumatic fractures in the incisive teeth. However, according to a recent Cochrane review, including 332 patients, early treatment using functional appliances reduced the incidence of associated traumas compared to late treatment protocol (Odds Ratio of $0.56,95 \%$ CI 0.33 to
0.95; moderate quality of evidence). ${ }^{40}$ In addition, the risk ratio assessment for new incisor trauma showed that offering early treatment reduced the risk of trauma by 33\% (in the functional group) and $41 \%$ (in the headgear group). Moreover, these authors stated that there was no marked advantage in two-phase treatment over singlephase treatment in adolescents, except where the teeth are so prominent that they are at higher risk of trauma. Thus, considering these particular situations, early interceptive treatment to reduce the existing overjet is recommended, rather than waiting to deliver a single-phase treatment. These authors based their statements on teeth alignment, forgetting the impact of a maxilla/mandible derangement on the growth and development of the craniofacial complex. Our results strongly indicated that early interventions on malocclusion have a considerable effect on the establishment/re-establishment of correct stomatognathic functions and, consequently, in the correct craniofacial growth pattern.

We assessed the quality of study evidence using standard protocols for risk of bias assessment and regular risk of bias domains within the Cochrane guidelines. Confidence in evidence was very low for all primary and secondary outcomes (craniofacial morphology and bone structures, masticatory system and functional trait, and impact of feeding preferences). Primarily, we downgraded the evidence due to methodological limitations of the studies (risk of bias across included studies), and imprecision (usually due to an insufficient number of enrolled patients, considering the elevated prevalence of malocclusion and distribution across populations). As far as publication bias is concerned, we mostly downgraded the evidence in this domain because retrieved studies appeared to emphasize only positive effects associated with the intervention. Previous research in the Dentistry field has already found a considerable number of studies "over estimating" the benefits of various interventions, including the field of 
Orthodontics. ${ }^{41,42}$ Therefore, the results synthesized in our review must be interpreted with caution, as a group of evidence is not always associated with generalized patterns of practical and clinical observations.

This systematic review has several strengths. Firstly, our review is based upon a considerable and representative number of databases, which precisely incorporates high-quality original studies. In addition, we carried out the systematic review following evidence-based medicine guidelines and adhered to an established/published protocol, with minor protocol deviations which did not jeopardize or impact the overall conclusions offered by the study. One protocol deviation is associated with the inclusion of participants outside the predefined age limit (only under six years old), essentially because we could not retrieve complete individual data from two specific studies. However, we believe that the inclusion of pediatric patients slightly older than six years of age might be acceptable and not harmful to the quality of the study as some authors have suggested that the deciduous dentition starts to shred between ages six and seven. ${ }^{43}$ Furthermore, we implemented a clearly defined search strategy and proceeded to high-standard methodological criteria/summary techniques. One potential limitation of our review relates to the fact that because different intervention protocols were used among included primary studies, we could not perform a meta-analytical assessment (pooling results into a forest plot graph). This has become a significant issue among methodologists and systematic review experts and is common when there is a considerable amount of missing information in included studies, along with differences in populations, interventions, comparisons, or methods that could make the average effect across studies meaningless. ${ }^{44}$ Nevertheless, we attempted to create a narrative summary of the findings table describing all potential effects from each implemented intervention. We believe that specialists across the world should focus and work collaboratively on the creation of randomized and adequately prepared trials to allow a better understanding of the associated effect between early intervention for malocclusion and craniofacial growth. Another potential limitation relies on the lack of access to 23 primarily eligible studies identified through the screening phase. However, because many of these titles were published before 1980, we believe that these studies' likelihood of meeting our inclusion criteria is low. Therefore, we are firmly convinced that the overall tendency of effect might not be compromised due to this limitation. Lastly, by excluding case reports and case series, we might have missed the opportunity to approach highly variable types of specific orthodontic/orthopedic interventions, probably used in a particular country, region of the dentistry office. However, by the transparent adhesion to our predefined protocol, the incorporation of such studies into our research is not feasible and thus, would not change the results presented hitherto, as well as would not interfere in the decision-making process, as we show here only studies with a minimum acceptable level of evidence.

\section{CONCLUSIONS}

In the last several years, the impact of early treatment of malocclusion has drawn the attention of oral-care specialists, in particular, because of the long-term consequences of the lack of delivery of appropriate therapy. This systematic review suggests that early interventions might improve facial asymmetry, along with increased palatal volume and palatal surface, leading to favorable sagittal growth of the maxilla. In addition, early interventions effectively interfere with the masticatory system, enhancing the average bite force magnitude and increasing the general thickness of facial muscles. Lastly, we observed a relevant correlation between the 
presence of malocclusion and infant feeding preference, particularly an irregular pattern of occlusion among those children under artificial feeding methodology. There is still a need for high-quality studies to explore the best treatment protocols for each type of malocclusion, contemplating patient-important outcomes.

\section{REFERENCES}

1. World Health Organization. Oral health [Internet]. Oral Health. [cited 2021 Apr 22]. Availablefrom:

https://www.who.int/westernpacific/healthtopics/oral-health

2. Perillo L, Esposito M, Caprioglio A, Attanasio S, Santini AC, Carotenuto M. Orthodontic treatment need for adolescents in the Campania region: the malocclusion impact on self-concept. Patient Prefer Adherence 2014;8:353-359

3. Akbari M, Lankarani KB, Honarvar B, Tabrizi $\mathrm{R}$, Mirhadi H, Moosazadeh M. Prevalence of malocclusion among Iranian children: A systematic review and meta-analysis. Dent Res J (Isfahan). 2016;13:387-395

4. Alvarado K, López L, Hanke R, Picón F, RivasTumanyan S. Prevalence of Malocclusion and Distribution of Occlusal Characteristics in 13- to 18-year-old Adolescents Attending Selected High Schools in the Municipality of San Juan, PR (2012-2013). Health Sci J 2017;36:61-66

5. Lombardo G, Vena F, Negri P, Pagano S, Barilotti C, Paglia L, Colombo S, Orso M, Cianetti $\mathrm{S}$. Worldwide prevalence of malocclusion in the different stages of dentition: A systematic review and meta-analysis. Eur J Paediatr Dent. 2020;21:115-122

6. Grippaudo C, Paolantonio EG, Antonini G, Saulle R, La Torre G, Deli R. Association between oral habits, mouth breathing and malocclusion. Acta Otorhinolaryngol Ita 2016;36:386-394

7.Kasparaviciene K, Sidlauskas A, Zasciurinskiene E, Vasiliauskas A, Juodzbalys G, Sidlauskas M, et al. The prevalence of malocclusion and oral habits among 5-7-year-old children. Med Sci Monit. 2014;20:2036-2042
8. Majorana A, Bardellini E, Amadori F, Conti G, Polimeni A. Timetable for oral prevention in childhood-developing dentition and oral habits: a current opinion. Prog Orthod 2015;16:39-48

9.Woon SC, Thiruvenkatachari B. Early orthodontic treatment for Class III malocclusion: A systematic review and meta-analysis. Am J Orthod Dentofacial Orthop 2017;151:28-52

10. Kallunki J, Bondemark L, Paulsson L. Outcomes of Early Class II Malocclusion Treatment - A Systematic Review. J Dent Oral Health Cosmesis 2018;3:009

11. Grippaudo C, Valerio P, Romeo P, Fiasca F, Quinzi V. Bite and Sight: Is There a Correlation? ClinicalAssociation between Dental Malocclusion andVisual Disturbances in Pediatric Patients. Appl Sci 2020;10: 5913-5920

12. Oka S, Kawanabe H, Yamanobe S, Fukui K, Baba Y, Deguchi T. Relationship between olfaction and maxillofacial morphology in children with malocclusion. Clin Exp Dent Res 2021;7(:33-39

13. Souto-Souza D, Soares MEC, Primo-Miranda EF, Pereira LJ, Ramos-Jorge ML, Ramos-Jorge J. The influence of malocclusion, sucking habits and dental caries in the masticatory function of preschool children. Braz Oral Res 2020;34:e059

14. Moher D, Liberati A, Tetzlaff J, Altman DG, PRISMA Group. Preferred reporting items for systematic reviews and meta-analyses: the PRISMA statement. PLoS Med 2009;6:e1000097

15. Sterne JA, Hernán MA, Reeves BC, Savović J, Berkman ND, Viswanathan M, et al. ROBINS-I: a tool for assessing risk of bias in non-randomised studies of interventions. BMJ 2016;355:i4919

16. Campbell M, McKenzie JE, Sowden A, Katikireddi SV, Brennan SE, Ellis S, et al. Synthesis without meta-analysis (SWiM) in systematic reviews: reporting guideline. BMJ 2020;368:16890

17. Kajiyama K, Murakami T, Suzuki A. Comparison of orthodontic and orthopedic effects of a modified maxillary protractor between deciduous and early mixed dentitions. Am J Orthod Dentofacial Orthop 2004;126:23-32

18. Carrascoza KC, Possobon R de F, Tomita LM, Moraes ABA de. Conseqüências do uso da mamadeira para o desenvolvimento orofacial em crianças inicialmente amamentadas ao peito. Jornal de Pediatria 2006;82:395-407 
19. Tollaro I, Baccetti T, Franchi L. Craniofacial changes induced by early functional treatment of Class III malocclusion. Am J Orthod Dentofacial Orthop. 1996 Mar;109(3):310-8.

20. Primozic J, Ovsenik M, Richmond S, Kau $\mathrm{CH}$, Zhurov A. Early crossbite correction: a three-dimensional evaluation. Eur J Orthod. 2009;31:352-356

21. Primožič J, Richmond S, Kau CH, Zhurov A, Ovsenik M. Three-dimensional evaluation of early crossbite correction: a longitudinal study. Eur J Orthod 2013;35:7-13

22. Castelo PM, Gavião MBD, Pereira LJ, Bonjardim LR. Evaluation of changes in muscle thickness, bite force and facial asymmetry during early treatment of functional posterior crossbite. J Clin Pediatr Dent 2010;34:369-374

23. Ferrer AL de, Villalba TBV de. Influencia de la succión deglución sobre el crecimiento y desarrollo orofacial. Rev Fac Cien Med Univ Nac Cordoba 2006;63:33-37

24. Huh A, Horton MJ, Cuenco KT, Raoul G, Rowlerson AM, Ferri J et al. Epigenetic influence of KAT6B and HDAC4 in the development of skeletal malocclusion. Am J Orthod Dentofacial Orthop. 2013;144:568-576

25. Valerio P, Perfeito F, Moura LP. Mandible protraction alters Type I collagen, osteocalcin and osteonectin gene expression in adult mice condyle. Ann Stomatol (Roma) 2018;8:95-103

26. Seelan, Ratnam S, Warner DR, Webb CL, Pisano M, Greene MR. Epigenetic regulation of Sox4 during palate development. Epigenomics 2013;5:131-146

27. Raterman, ST, Metz JR, Wagener FADTG, Hoff JW. Zebrafish Models of Craniofacial Malformations: Interactions of Environmental Factors. Front Cell Dev Biol 2021;9:650948

28. Liberton DK, Verma P, Almpani K, Fung PW, Mishra R, Oberoi S, et al. Craniofacial Analysis May Indicate Co-Occurrence of Skeletal Malocclusions and Associated Risks in Development of Cleft Lip and Palate. J Dev Biol 2020;8:2-11

29. Doraczynska-Kowalik A, Nelke KH, Pawlak W, Sasiadek MM, Gerber H. Genetic Factors Involved in Mandibular Prognathism. J Craniofac Surg 2017;28:422-431

30. Campbell M, McKenzie JE, Sowden A, Katikireddi SV, Brennan SE, Ellis S et al.
Synthesis without meta-analysis (SWiM) in systematic reviews: reporting guideline. BMJ 2020;368:16890

31. Musich, DR, Busch, MJ. Early Orthodontic Treatment: Current Clinical Perspectives. Alpha Omegan 2007;100:17-24

32. Fleming C. Ejection problems and injuries: their causes, effects and treatments, and suggestions for preventive measures. Aviat Space Environ Med 1979;50:829-833

33. Kluemper GT, Beeman CS, Hicks EP. Early orthodontic treatment: what are the imperatives? J Am Dent Assoc 2000;131:613-620

34. Scheffel DLS, Jeremias F, Fragelli CMB, Dos Santos-Pinto LAM, Hebling J, de Oliveira OB Jr. Esthetic dental anomalies as motive for bullying in schoolchildren. Eur J Dent 2014;8:124-128

35. Bagley H, Gorst S, Morley R, Young B. Using patient-important outcomes for systematic reviews [Internet] [cited 2021 Apr 22]. Available from: https://uk.cochrane.org/news/usingpatient-important-outcomes-systematic-reviews

36. Gaudry S, Messika J, Ricard J-D, Guillo S, Pasquet B, Dubief E, et al. Patient-important outcomes in randomized controlled trials in critically ill patients: a systematic review. Ann Intensive Care 2017;7:28-35

37. Fleming PS. Timing orthodontic treatment: early or late? Aust Dent J 2017;62:11-19

38. Kiyak HA, Haluk I, Miotti FA. Orthodontists' perspectives regarding treatment timing: a crossnational study. World J Orthod 2004;5:40-47

39. Keim RG, Gottlieb EL, Nelson AH, Vogels DS 3rd. 2013 JCO Orthodontic Practice Study. Part 1: trends. J Clin Orthod. 2013 Nov;47(11):661-80. PMID: 24605392.

40. Batista KB, Thiruvenkatachari B, Harrison JE, O'Brien KD. Orthodontic treatment for prominent upper front teeth (Class II malocclusion) in children and adolescents PubMed. Cochrane Database of Systematic Reviews 2018;3:CD003452.

41. Murad MH, Chu H, Lin L, Wang Z. The effect of publication bias magnitude and direction on the certainty in evidence. BMJ Evid Based Med. 2018;23:84-86

42. Koletsi D, Fleming PS, Eliades T, Pandis N. The evidence from systematic reviews and metaanalyses published in orthodontic literature. Eur J Orthod 2015;37:360-369 
43. American Dental Association. Tooth eruptionThe primary teeth. [Internet] [cited 2021 June 11] Availablefrom:

https://www.ada.org/ /media/ADA/publicatio ns/Files/patient_56.pdf

44. Cochrane Effective Practice and Organisation Care. Synthesising results when it does not make sense to do a meta-analysis [Internet] [cited 2021 June 11]Cochrane Group; Available from: https://epoc.cochrane.org/sites/epoc.cochrane.o $\mathrm{rg} /$ files/public/uploads/Resources-forauthors2017/synthesising_results_when_metaanalysis_does_not_make_sense.pdf

\section{How to cite this article:}

Patrícia Valério, Tina Poklepović Peričić, Andrea Rossi, Cristina Grippaudo, Júlia dos Santos Tavares Campos, Israel Júnior Borges do Nascimento. The effectiveness of early intervention on malocclusion and its impact on craniofacial growth: a systematic review. Contemp Pediatr Dent 2021:2(2):1-18. DOI: $\underline{10.51463 / \mathrm{cpd} .2021 .61}$

\section{Declarations}

Acknowledgements: Not applicable.

Conflict of Interest Statement: The authors disclose no potential conflicts of interest.

Ethics Statement: This systematic review is reported following the Preferred Reporting Items for Systematic review and Meta-analyses (PRISMA) and was registered in the PROSPERO (CRD42021226182).

Informed Consent: Not applicable.

Author contributions: Conception and design: All Authors; Acquisition of data: All Authours; Interpretation of data: All Authors; Drafting article: PV TPP, IJBN; Revision artice: PV IJBN; Final approval: All Authors

Funding: This work is not finantiated.

Data Availability: The data used to support the findings of this study can be made available upon request to the corresponding author.

Peer-review: Externally double-blinded peer-reviewed. 